\title{
Process intensification and process control in freeze-drying
}

\section{Barresi, A. A.*; Pisano, R.}

Department of Applied Science and Technology. Politecnico di Torino, Torino, Italy

*E-mail of the corresponding author: antonello.barresi@polito.it

\begin{abstract}
Widespread use of advanced process control allows reduction of costs, by reducing drying time and energy consumption. The "control of the freezing stage" (by forced nucleation) also appears to be beneficial to process intensification, as it can impact the product structure and modify the product resistance to mass transfer. An alternative way to increase the drying rate is the use of organic solvents as they can lead to larger solvent crystals, hence lower product resistance to vapor flow.

Atmospheric freeze-drying may be a good alternative to vacuum freezedrying, as a way of increasing process efficiency. A further improvement can be obtained by combining atmospheric or vacuum freeze-drying with new technologies.

A further step towards process intensification is given by continuous plants, as this allows a dramatic increase in throughput and product quality uniformity.
\end{abstract}

Keywords: freeze-drying; process intensification; controlled nucleation; continuous process. 


\section{Introduction}

The freeze-drying process is widely used in pharmaceuticals manufacturing, as well as for drying foodstuffs, as high-quality products can be obtained due to the low operating temperatures. Anyway it is quite an expensive process, and this fact limits applications in food and probiotic industry. Reduction of costs, by reducing drying time and energy consumption, and a better uniformity in end-product quality can be reached by means of process optimization and widespread use of advanced process control, but there is a big potential for process intensification by process modifications.

According to Stankiewicz and Moulijn [1], process intensification can be obtained by modifying equipment, to optimize critical parameters (e.g., heat transfer and mass transfer), or by process intensifying methods, changing the process or using alternative energy sources. Proposed control approaches, and in particular advanced process control, will be first discussed in this review. Other possibilities for process intensification will be then discussed, focusing in particular on controlled nucleation and process modifications in production of pharmaceuticals.

\section{Process optimization and widespread use of advanced process control}

Improvement of the process control has been recognized as a development need for the pharmaceutical industry over the last thirty years, but relatively few changes occurred at the production scale. It must be evidenced that today even the most advanced industrial freezedryers have no robust process control. An open-loop control approach is generally adopted, but rarely the cycle has been really optimized, and cycle transfer between different pieces of equipment or scale up is generally challenging [2-5].

Effective monitoring and control systems are required to manage the process in such a way that product quality is not jeopardized at the end of the process. Besides, as the duration of the freeze-drying process is an important concern, the control systems should be able to optimize in-line the operating conditions, namely the pressure in the drying chamber and the heating power, i.e. the temperature of the heating source. In the following different the methods proposed will be analyzed.

A first group of methods is based on the measurement of product temperature obtained through a thermocouple (or another temperature sensor); they optimize only the temperature of the heating element. In this case it is possible to use just the temperature measurement, with a fuzzy logic-based algorithm. Alternatively, a mathematical model can be coupled to the experimental measurements, thus obtaining a soft-sensor; this can be used 
to calculate the optimal control actions using, for example, a PI algorithm, or calculating in line the design space of the process [6-8].

The second group of methods is based on the pressure rise test [9]; this is a technique for the in-line process identification that allows estimating both the state of the product (temperature and residual amount of ice) and the values of some model parameters. By this way a mathematical model can be used to optimize the process using, for example, a classic proportional algorithm, or an advanced Model Predictive Control algorithm [10,11]. Control logics based on measurement of heat or sublimation flux will be also discussed.

Control procedures are available also for the secondary drying step. The key feature of the method is the coupling of the measurement of the desorption rate, obtained by means of the PRT or other devices, with a mathematical model of the process [12].

\section{Control of the freezing stage}

Freezing plays a fundamental role in the freeze-drying process, as it determines product morphology and, therefore, drying performances and drug stability [13]. Therefore, it has become essential to increase the knowledge and control over this process. A brief overview of the tools which allow control of the impact of freezing on product morphology, and, consequently, on drying efficiency and product quality will be presented.

During freezing, the stochastic nature of nucleation is regarded as a demerit, because it is directly linked to vial-to-vial variability in terms of product morphology, drying behavior and, ultimately, product stability. Hence, lots of attention was given to the control of the temperature at which nucleation occurs, trying at least to make it as uniform as possible over a batch of vials. In fact, it is not actually possible to really "control the nucleation" even if this term is often used for simplicity; as said before, what is possible to do, is to force nucleation to take place at a given temperature and control the batch temperature holding it until nucleation is completed. The higher the nucleation temperature, the larger the ice crystal size, and thus the lower the cake resistance. In recent years, almost all the principal freeze-dryers manufacturers developed their proprietary technology to induce ice crystallization and to reduce the time span for completing the freezing in all the vials of the batch. Many patents were deposited, and different technical solutions were made commercially available, especially at the pilot-scale; even if many difficulties still limit somehow the application to large production scales of some methods, controlled ice nucleation starts moving also into manufacturing [14,15].

Ultrasound nucleation has been the object of an extensive research, but the passage from lab to commercial scale faced strong difficulties. The main problems were related to the difficulty to efficiently propagate the ultrasonic waves, and to scale the process, as the 
optimum ultrasonic frequency depends on the set up. Actually commercially available equipment adopts either a variant of the ice fog, or the depressurization method, or the vacuum induced nucleation. The ice fog concept is probably the first method proposed to control nucleation (as it is known since 1990), but only recently the technical developments made it really suitable for practical applications.

The easiest and cheapest way to control nucleation is surely the vacuum induced surface freezing, VISF (also known as vacuum induced nucleation, VIN), as this technology requires no hardware, any equipment can be easily retrofitted, and there are limited sterility concerns (related to sterilization of large valves required for rapid depressurization) [16]. It is important to evidence that notwithstanding the concern for possible product denaturation, in particular with the depressurization and the VIN method (for the possible presence of small bubbles), all the discussed controlled nucleation technologies gave particularly good results; in fact, as a consequence of the larger ice crystals (and thus of their reduced solid surface), an improved stability even for very sensitive products was observed.

Thus the described methods are effective approaches to process intensification, but to guarantee the required final quality and uniformity of the batch, they must be coupled with methods that assure the temperature uniformity of all the vials. In all cases, the depressurization rate is the element that may limit the applicability to large units, as it poses constrains to the geometric characteristics of the apparatus and of the depressurization circuit.

\section{Process modifications}

The use of a strictly organic or organic/water system is beneficial to both product quality and process optimization. Potential advantages and disadvantages of use of organic solvents and cosolvents (mixed with water, that up to now has been the most common solvent used) in freeze-drying are widely discussed by Teagarden and Baker [17]. The main advantage in addition to the increase in solubility of the product, is the increase in rate of sublimation and hence decrease of drying time. However, before a specific solvent is used in the manufacture of a parenteral product, lyophilization professionals have to carefully weigh advantages and disadvantages.

The use of organic liquids, either as solvent or co-solvent, produces larger ice crystals and, thus, increases the average diameter of the pores created during ice sublimation. As a result, the product resistance to vapor flow decreases and, if product temperature does not change, the rate of sublimation increases, as said above. In addition to the reduction in product resistance, most of the cosolvents used in freeze-drying applications increase the rate of sublimation because they have a higher vapor pressure than water and, hence, they increase the driving force for mass transfer. In addition, a further reduction in energy consumption 
results from the fact that the sublimation enthalpy of organic solvents is smaller than that of water ice. Unfortunately, organic solvents are rarely used in the manufacture of a lyophilized pharmaceutical product, mainly because of safety concerns. Specifically, attention has to be paid to how the organic solvents can be safely handled, e.g., preventing fires or explosions during their manipulation or avoiding contamination of the vacuum pump oil, which can decrease the pump efficiency and, hence, impede adequate control of pressure inside the equipment. Furthermore, in the case of mixture of solvents, monitoring the state of progress of drying might be very complex, because the various solvents can show significantly different rates of sublimation. Therefore, it is necessary to track the separation of the individual solvents, e.g., using sensors that are sensitive to only specific solvents and completely insensitive to others [18].

In food processing, vacuum freeze-drying produces dried products that retain almost all their original characteristics, e.g. color, flavor, and taste. The high specific surface area generally allows an easy and fast rehydratation. The drawback is represented by the cost of the operation: fixed costs can be high due to vacuum requirement, and the energy cost can be significantly higher with respect to other drying processes (the specific moisture extraction rate in a vacuum freeze-drying process is in the range of $0.4 \mathrm{~kg}$ of water per $\mathrm{kWh}$ ). In order to reduce the cost and the energy consumption of the process, thus improving its sustainability, the atmospheric freeze-drying (drying with cold air or nitrogen at normal pressure) has been proposed [19,20]: in this case it is possible to achieve a specific moisture extraction rate ranging from 1.5 to $4.6 \mathrm{~kg}$ of water per $\mathrm{kWh}$. For example it has been claimed that up to $35 \%$ of energy savings could be achieved when using atmospheric freeze-drying instead of vacuum freeze-drying for potato slices.

Most of the literature deals with atmospheric freeze-drying in fluidized bed dryers and in spray freeze-dryers. When the atmospheric freeze-drying is carried out in a fluidized bed it can take advantage of the high values of the heat and mass transfer coefficients; the product has to be frozen and granulated before drying. A drawback of the process is represented by the size reduction caused by mechanical cracking. As an alternative it is possible to carry out the process in a tunnel dryer, even if the heat and mass transfer is not so good.

A detailed comparison of vacuum and atmospheric freeze-drying has not been carried yet, and work is currently ongoing also in the labs at Politecnico di Torino. Surely drawbacks related to the limitation in process temperature, to avoid ice melting, and the necessity to recirculate and dry large volumes of gas at low temperature must be taken into account. In particular, atmospheric freeze-drying produces higher quality products (in comparison to traditional drying) but still entails long drying times. Energy consumption can be reduced by new technologies which use alternative forms and sources of energy for processing. These technologies can be applied to either enhance heat transfer between product and heat source, such as microwave, radiofrequency and infrared radiation, or simply intensify the rate of dehydration without increasing the amount of heat supplied to the product, e.g. by 
using high intensity sonic and ultrasonic waves.

Spray freeze-drying seems to be a valuable alternative to produce a free-flowing powder, with high surface area, porous end product, and good instant characteristics, with enhanced solubility and a uniform and ultrafine particle size. Spray freeze-drying into liquids, gases (e.g. a refrigerated air stream), and into gases over a fluidized bed have been reported in the literature. This technology has also a good potential for continuous processes. Spray freezedrying allows the massive production of pharmaceuticals for dry powder inhalation, and a more precise control of particle size compared to spray drying. Furthermore, the rapid cooling rates promote the formation of glassy water that is beneficial in preventing the aggregation of proteins during the cryo-concentration phase.

\section{Continuous plants}

Recently, many pharmaceutical manufacturers are trying to convert their processes in favor of continuous production. To achieve this objective, it is necessary to integrate those production steps, that are performed sequentially in a conventional batch configuration, in a continuous process, leading to more compact units with a higher degree of automation and fewer manual interventions. This is particularly true for the lyophilization of pharmaceuticals and biopharmaceuticals in unit-doses which, although it is a robust and well-established technology, still remains inefficient and expensive. For example, the drying behavior within a batch, as well as from batch to batch, is still a problem of deep concern, despite the elaborate equipment design and the sophisticated control systems recently introduced.

It must be noted that continuous freeze-drying find currently application mainly for soluble coffee production. Many patents have been deposited for food technology applications, but realizations are still very limited. In the pharmaceutical industry there are much more severe constrains, and just a relatively few patents have been deposited. In particular, so far, only two technologies have been proposed for the production of end-to-use lyophilized products in a continuous way [21,22]. In both technologies, processing time and equipment size is dramatically reduced, up to 10 times, and all manual interventions and breaks have been minimized reducing the risk of product contamination. Furthermore, in-line control can easily be implemented, and scale-up simply consists of adding parallel modules. Despite their numerous advantages, the application of these technologies to real cases in industry still requires time. The above proposed technologies are still in development, and their capability to work under GMP conditions, meeting all the stringent requirements of a pharmaceutical production, has not been completely demonstrated yet. Nevertheless, these solutions are concrete steps toward continuous manufacturing of lyophilized pharmaceuticals, similarly to what food industry did years ago. 


\section{Conclusions}

Different approaches to process intensification, coupled with improved process control for freeze-drying have been discussed. In the last years, many progress have been carried out in process monitoring and control, including the control of the freezing step. Current research should favor the transition towards a more robust design of freeze-drying cycles, based on deep knowledge of phenomena involved rather than on empirical observations. This would be extremely beneficial, especially for the pharmaceutical industry, where particular emphasis is placed on product homogeneity and process control. Process modifications, like atmospheric freeze drying and spray-freeze drying are interesting alternatives whose real potentiality are currently under investigation. The most ambitious goal is certainly the realization of a continuous process at the industrial scale for pharmaceuticals.

The intensification of the process and the reduction of the processing costs, on the other way, may open new possibilities of wider application of freeze drying in processing of valuable foods and in particular of probiotics, for which the market is requiring higher qualitative standards.

\section{References}

[1] Stankiewicz, A.J.; Moulijn, J.A. Process intensification: Transforming chemical engineering. Chemical Engineering Progress 2000, 96, 22-34.

[2] Nail, S.; Gatlin, L.A. 1985. Advances in control of production freeze dryers. PDA Journal 1985, 39 (1), 16-27.

[3] Liapis, A. I., Pikal, M. J. Bruttini, R. Research and development needs and opportunities in freeze drying. Drying Technology 1996, 14 (6), 1265-1300.

[4] Sadikoglu, H.; Ozdemir, M.; Seker, M. Freeze-drying of pharmaceutical products: Research and development needs. Drying Technology 2006, 24, 849-861.

[5] Fissore, D.; Pisano, R.; Barresi, A.A. A model based framework to optimize pharmaceuticals freeze-drying. Drying Technology 2012, 30, 946-958.

[6] Fissore, D.; Pisano, R.; Barresi, A.A. On the use of temperature measurement to monitor a freeze-drying cycle for pharmaceuticals. In Proceedings of IEEE International Instrumentation and Measurements Technology Conference "I2MTC 2017”, Torino, Italy, May 22-25, 2017; 1276-1281.

[7] Bosca, S.; Barresi, A.A; Fissore, D. On the use of model-based tools to optimize inline a pharmaceuticals freeze-drying process. Drying Technology 2016, 34 (15), 1831-1842.

[8] Bosca, S.; Barresi, A.A.; Fissore, D. On the robustness of the soft-sensors used to monitor a vial freeze-drying process. Drying Technology 2017, 35 (9), 1085-1097. 
[9] Fissore, D.; Pisano, R.; Barresi, A.A. On the methods based on the Pressure Rise Test for monitoring a freeze-drying process. Drying Technology 2011, 29 (1), 73-90.

[10] Pisano, R.; Fissore, D.; Barresi, A.A. Freeze-drying cycle optimization using Model Predictive Control techniques. Industrial \&. Engineering Chemistry Research 2011, 50 (12), 7363-7379.

[11] Pisano, R.; Fissore, D.; Barresi A.A. In-line and off-line optimization of freeze-drying cycles for pharmaceutical products. Drying Technology 2013, 31 (8), 905-919.

[12] Fissore, D.; Pisano R.; Barresi, A.A. Monitoring of the secondary drying in freezedrying of pharmaceuticals. Journal of Pharmaceutical Science 2011, 100 (2), 732742.

[13] Kasper, J.C.; Friess, W.F. The freezing step in lyophilization: Physico-chemical fundamentals, freezing methods and consequences on process performance and quality attributes of biopharmaceuticals. European Journal of Pharmaceutics and Biopharmaceutics 2011, 78 (2), 248-263.

[14] Thomas, P. Controlled ice nucleation moves into manufacturing. Pharmaceutical Manufacturing 2011, article 20.

[15] Geidobler, R.; Winter, G. Controlled ice nucleation in the field of freeze-drying: Fundamentals and technology review. European Journal of Pharmaceutics and Biopharmaceutics 2013, 85 (2), 214-222.

[16] Arsiccio, A.; Oddone, I.; Barresi, A.A.; Van Bockstal, P.-J.; De Beer, T. Vacuum Induced Surface Freezing as an effective method for improved inter- and intra-vial product homogeneity. European Journal of Pharmaceutics and Biopharmaceutics 2018 (in press). DOI: 10.1016/j.ejpb.2018.04.002

[17] Teagarden, D. L.; Baker, D. S. Practical aspects of lyophilization using non-aqueous co-solvent systems. European Journal of Pharmaceutical Science 2002, 15 (2), 115133.

[18] Fissore, D.; Pisano, R.; Barresi, A.A. Process analytical technology for monitoring pharmaceuticals freeze-drying - A comprehensive review. Drying Technology 2018 (in press). DOI: 10.1080/07373937.2018.1440590

[19] Claussen, I.C.; Ustad, T.S.; Strømmen, I.; Walde, P.M. Atmospheric freeze drying A review. Drying Technology 2007, 25 (6), 957-967.

[20] Fissore, D.; Coletto, M.; Barresi A.A. Atmospheric food freeze-drying: Challenges and opportunities. New Food 2013, 16 (5), 11-14.

[21] Corver, J.A.W.M. Method and system for freeze-drying injectable compositions, in particular pharmaceutical compositions. US Patent 20140215845, 2012.

[22] Pisano, R.; Capozzi, L.C.; Trout, B.L. Continuous freeze-drying and its relevance to the pharma/biotech industry. In Integrated Continuous Biomanufacturing III, Farid S. Alves P., Warikoo V. (eds.), ECI Symposium Series, 2017. http://dc.engconfintl.org/biomanufact_iii/70 